\title{
Analysis on Integration of Wave Energy Converter Systems
}

\author{
Chao Liu ${ }^{1}$, Jing Wang ${ }^{2}$ \\ TianJin University of Technology and Education, Tianjin, China ${ }^{1}$ \\ TianJin University of Technology and Education, Tianjin, China ${ }^{2}$
}

\begin{abstract}
For the past few years, interest in wave energy is growing very fast. The ocean holds massive amount of clean and untouched energy, which can show an important role in supplying our energy demand. It is approximated that total energy from the coast of the United States is 252 million MWh/year. Oceans and sees around the world contain plentiful amount of energy that exceeds today's power generation needs. Wave Energy Converters (WEC) are devices to convert wave energy into electrical energy. One example of WEC technology is Oscillating Water Columns (OWCs). OWCs are devices with a semi-submerged chamber or hollow open to the sea below, keeping a trapped air pocket above a water column. Waves force the column to act like a piston, moving up and down, forcing the air out of the chamber and back into it. This continuous movement force a bidirectional stream of high-velocity air, which is channelled through a Power-Take-Off (PTO). The PTO system converts the airflow into energy. This paper discusses different types of wave energy converters and challenges regarding their integration to power grid.
\end{abstract}

Keywords: Storage Units, Wave Energy, Dc Voltage

\section{INTRODUCTION}

The traditional energy network has been undergoing major changes. The penetration of distributed renewable energy generation has been prompted by several factors, such as environmental issues, market deregulation, incentive policies and growth in the global demand for electricity. The benefits of distributed renewable energy generation can include reliability enhancement, power loss reduction, improvement in power quality, the integration of renewable sources and the provision of ancillary services [1]. However, if not addressed properly, increasing penetration of distributed energy resources in the power system, can also have negative effects on power system voltage control and accumulative communication and control delays of different parts, can lead to frequency instability [2]. It is in this context that microgrids arise as a platform where distributed renewable energy generation technologies can be readily integrated into the distribution network.

For the past few years, interest in wave energy is growing very fast. The ocean contains huge amount of clean and untouched energy, which can show an important role in supplying our energy demand [3-6]. It is approximated that total potential energy from the coast of the United States is 252 million MWh/year. Oceans and sees around the world contain plentiful amount of energy that exceeds today's power generation needs. There are different ways of using such renewable resources to make use of them in the most optimum way which results in reduction of total costs that should be paid by government [7]. Wave energy converters (WEC) are devices to convert wave energy into electrical energy. One example of WEC technology is point-absorber, which consists of a floating body on sea surface, and a cable that connects buoy to the electric generator on seafloor. The buoy will move along vertically with wave motion, and the electrical generator converts vertical motion of buoy into electric energy.

Based on their energy conversion mechanism, wave energy conversion systems can be categorized into four main groups of oscillating water column, attenuator, overtopping, and point absorbers. These different topologies of WECs are shown in Figs 1-4. Among these technologies, point absorbers seem superior due to lower cost, higher efficiency and reliability. Common point absorber approaches employ either a linear electric generator (or direct-drive) or a common rotary electric generator. As in the first case the vertical motion of buoy is directly connected to translator of linear electric generator, it is called direct-drive, while in latter case there is need for a medium apparatus to convert the linear vertical motion of buoy into rotary motion of electric generator. The rotary generator approach suffers from increased cost, volume and maintenance of the system. Linear generators, on the other hand, have fewer and reduced maintenance. Hence, direct-drive WEC (DDWECs) are promising solutions for wave energy conversion systems. One example of WEC technology is Oscillating Water Columns (OWCs). OWCs are devices with a semi-submerged chamber or hollow open to the sea below, keeping a trapped air pocket above a water column. Waves force the column to act like a piston, moving up and down, forcing the air out of the chamber and back into it. This continuous 


\section{International Advanced Research Journal in Science, Engineering and Technology}

ISO 3297:2007 Certified

Vol. 5, Issue 8, August 2018

movement force a bidirectional stream of high-velocity air, which is channelled through a Power-Take-Off (PTO). The PTO system converts the airflow into energy.

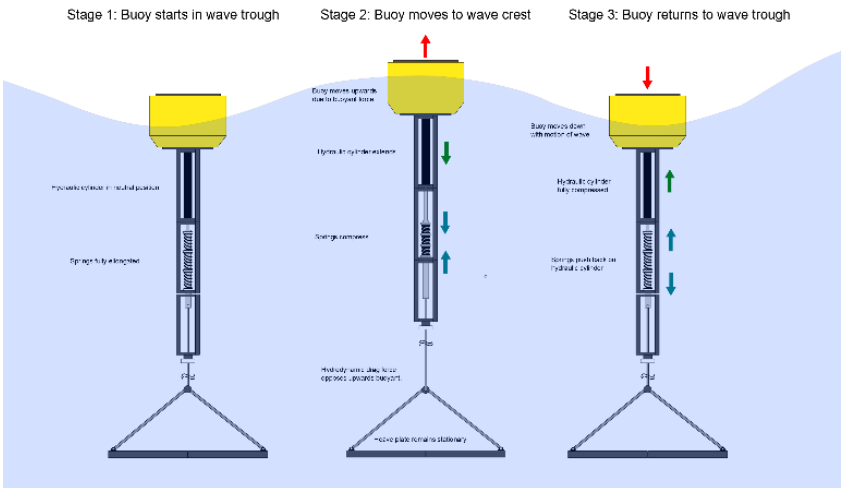

Fig. 1 Buoy operation for absorbing wave energy

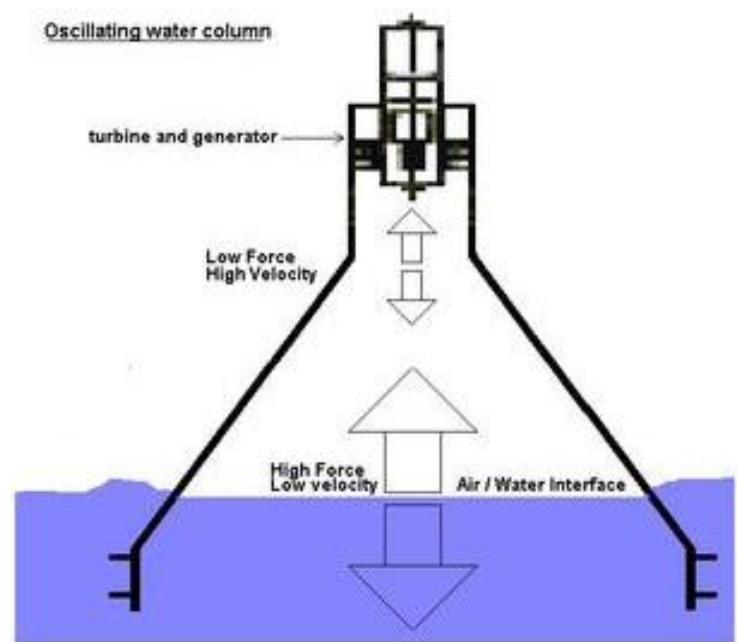

Fig. 2 oscillating water column wave energy system

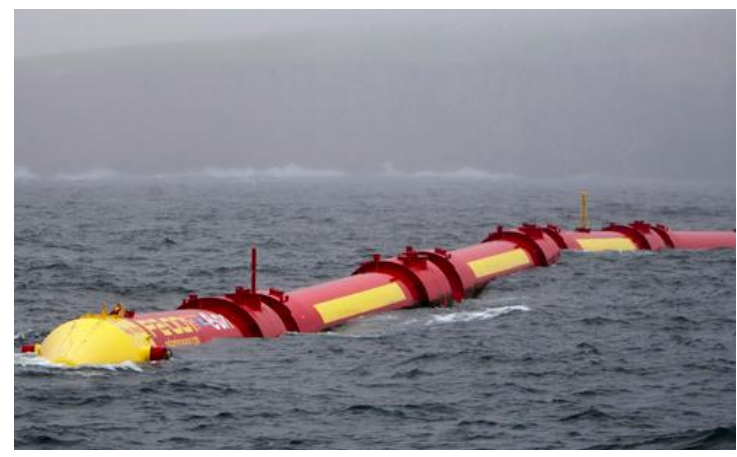

Fig. 3 attenuator wave energy converter

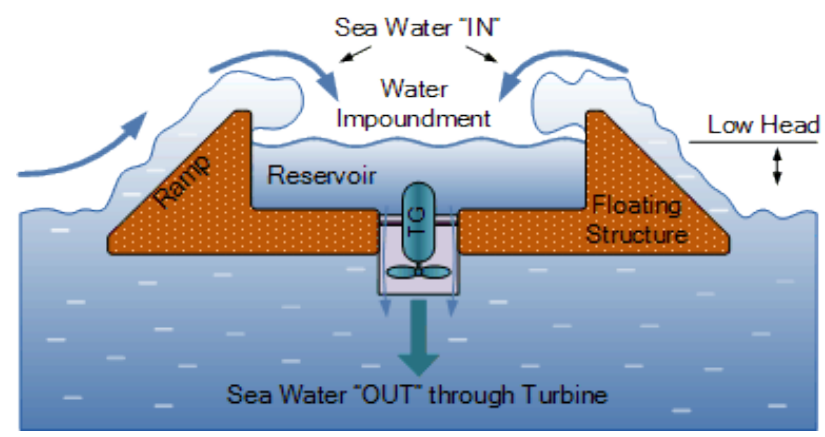

Fig. 4 Overtopping wave energy converter 


\section{International Advanced Research Journal in Science, Engineering and Technology \\ ISO 3297:2007 Certified}

Vol. 5, Issue 8, August 2018

The output active power of direct-drive linear WECs is extremely changing. With the vertical movement of the ocean waves and point absorbers, the movable part of the LPMG also moves vertically. Hence, both the magnitude and direction of velocity of the movable part of LPMG will have variations. The mechanical energy is changing into electrical energy by means of different kinds of electrical machines such as permanent magnet synchronous generator (PMSG), double fed induction generator (DFIG), dual mechanical port machines (DMPM), and so on [8]. The output power of DDWEC will be maximum in a certain moment and then gets to zero in few seconds after. The goal of applying an energy storage is to regulate the WEC output active power, so that it could be transmitted into grid.

\section{INTEGRATION OF WAVE POWER TO GRID INCORPORATING STORAGE}

Energy storage devices are great solution for smoothing the output power fluctuations of renewable energy resources and incorporating them to the grid, as shown in Fig. 5. So, there is need for an energy storage system to work in parallel with the main renewable energy source for connecting the renewable energy source to the power grid. The operation of the energy storage system is as follow: when the generated power of renewable source is higher than the power to be delivered to grid, the excess power is stored in the energy storage device; and when the generated power of renewable source is less than the power to be delivered to the grid then deficit is provided by the energy storage system Installing grid size battery is usually expensive and counted as one of the main drawback of integrating renewable into the grid. Recently, virtual batteries are introduced as a novel tool to aggregate and coordinate the large number of flexible residential resources and provide a synthetic reserve to grid operator to ensure the stability. However, these sources are flexibility are not completely controllable. Therefore, to benefit the most from them and to ensure stability, these resource should be dispatched robustly [9].

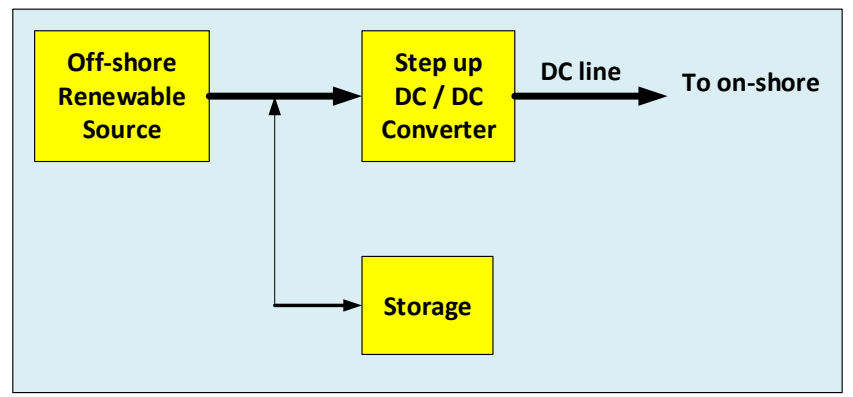

Fig. 5 Configuration of renewable energy source integration.

\section{MEDIUM-VOLTAGE STEP UP DC / DC CONVERTERS}

In recent years, industry has begun to demand higher power equipment, which now reaches the megawatt level. Controlled ac drives in the megawatt range are usually connected to the medium-voltage network. Today, it is hard to connect a single power semiconductor switch directly to medium voltage grids $(2.3,3.3$, 4.16, or $6.9 \mathrm{kV})$. Also, renewable energy sources are an important option for future power demand due to an increased concern for the environment. Trends toward large-scale integration and high-power application of green energy resources necessitate the advent of efficient power converter topologies, such as multilevel converters, with enhanced characteristics such as capability of handling voltage and power in the range of several $\mathrm{kV}$ and $\mathrm{MW}$, respectively. For these reasons, a new family of multilevel inverters has emerged as the solution for working with higher voltage levels.

Medium-voltage dc and high-voltage dc grids have been the focus of many research studies recently regarding their increasing applications in rapidly growing grid-connected renewable energy systems, such as wind, solar and wave farms [10-15]. To connect dc renewable resources to the grid Voltage Sourced Converters (VSC) are used commonly [16]. Moreover, VSC's can be used in MVDC and HVDC systems to connect ac sources to the dc bus [16]. Due to higher efficiency and flexibility, the MVDC systems are being considered as alternatives to ac distribution networks. Dc transformer is the key component for electrical isolation and voltage matching purposes in MVDC grids. In medium-voltage ac (MVAC) systems, line-frequency transformers are employed for stepping up/down the voltage. In MVDC grids, however, dc/dc converters are widely being utilized for those purposes

\section{DISCUSSION AND CONCLUSION}

Energy storage deployments in emerging markets worldwide are expected to grow over 40 percent annually in the coming decade, adding approximately $80 \mathrm{GW}$ of new storage capacity to the estimated 2 GW existing today. Application of Energy storage in power system will make the sending energy to the customers more reliable and economical. The energy will be stored while the electricity price is low and released back into the power network in 
peak power demand time (while the electricity price is high) [17]. Some research has also been done on the application of the Internet of Things (IoT) on using some household appliances as virtual storage. For instance, it was shown in [18] that $1.5 \mathrm{GW}$ of virtual storage capacity can be provided by having a control over swimming pool pumps of the state of Texas in the US

With the growing importance of renewable energy sources, scientist and engineers are anxious to enhance efficiencies and to lower the costs of these storage technologies. Yet, there seems to be only a handful of technologies available that are efficient enough and economical. Storing energy is not an easy task, as most of us know. Our smart phone battery only lasts for about a day laptops only a few hours; the range for electric cars is limited to only little more than a 100 kilometers; and these are only examples for comparatively small devices. Now imagine the problem of storing energy at the level of hundreds to thousands of wind turbines and photovoltaic cells. Employing the storage systems require knowledge on the generation type, load management, and requirements of the storage system for wave energy application

\section{REFERENCES}

[1] M. S. Modarresi and L. Xie, "An operating reserve risk map for quantifiable reliability performances in renewable power systems," 2014 IEEE PES General Meeting, National Harbor, 2014, pp. 1-5.

[2] Amini, Mahraz, and Mads Almassalkhi. "Investigating delays in frequency-dependent load control." Innovative Smart Grid Technologies-Asia (ISGT-Asia), 2016 IEEE. IEEE, 2016.

[3] M. Amundarain, M. Alberdi, A. J. Garrido and I. Garrido, "Modeling and simulation of wave energy generation plants: Output power control", IEEE Trans. Ind. Electron., vol. 58, no. 1, pp. 105-117, Jan. 2011.

[4] A. F. de O. Falcao, "Wave energy utilization: a review of technologies," Renewable and Sustainable Energy Reviews, vol. 14, no. 3, pp. 899918, Apr. 2010.

[5] S. Jafarishiadeh, M. Farasat "Modeling and Sizing of an Undersea Energy Storage System", IEEE Transactions on Industry Applications, vol. 54, no. 3, pp. 2727-2739, 2018.

[6] R. Ekström, B. Ekergård, and M. Leijon, "Electrical damping of linear generators for wave energy converters - a review", Renewable and Sustainable Energy Reviews, vol 42, pp. 116-128, Feb. 2015.

[7] N. Ghanbari; H. Mokhtari; S. Bhattacharya, "Optimizing Operation Indices Considering Different Types of Distributed Generation in Microgrid Applications", Energies 2018, 11, 894.

[8] Mohammad Ghanaatian; Ahmad Radan " Modeling and simulation of Dual Mechanical Port machine" 4th Annual International Power Electronics, Drive Systems and Technologies Conference 2013.

[9] Amini, Mahraz, and Mads Almassalkhi. "Trading off robustness and performance in receding horizon control with uncertain energy resources." Power Systems Computation Conference (PSCC). 2018.

[10] S Jafarishiadeh, M Farasat, AK Sadigh "Medium-voltage DC grid connection using modular multilevel converter," in Proc. Energy Conversion Congress and Exposition (ECCE), Cincinnati, OH, USA, pp. 2686-2691, 2017.

[11]Ferreira, J. A.: 'The multilevel modular dc converter', IEEE Trans. Power Electron., 2013, 28, (10), pp. 4460-4465.

[12] Chen, W., Huang, A. Q., Li, C., et al.:'Analysis and comparison of medium voltage high power DC/DC converters for offshore wind energy systems, IEEE Trans. Power Electron., 2013, 28, (4), pp. 2014-2023

[13] S. Jafarishiadeh, V. Dargahi, AK. Sadigh, M. Farasat, "Novel multi-terminal MMC-based de/dc converter for MVDC grid interconnection," IET Power Electronics, vol. 11, no. 7, pp. 1266-1276, June 2018.

[14] Engel, S. P., Stieneker, M., Soltau, N., et al.:'Comparison of the modular multilevel DC converter and the dual-active bridge converter for power conversion in HVDC and MVDC grids, IEEE Trans. Power Electron., 2015, 30, (1), pp. 124-137

[15] S. Jafarishiadeh, M. Farasat, S. Mehraeen "Grid-connected operation of direct-drive wave energy converter by using HVDC line and undersea storage system" in Proc. Energy Conversion Congress and Exposition (ECCE), Cincinnati, OH, USA, pp. 5565-5571, 2017.

[16]P. M. Shabestari, S. Ziaeinejad and A. Mehrizi-Sani, "Reachability analysis for a grid-connected voltage-sourced converter (VSC)," 2018 IEEE Applied Power Electronics Conference and Exposition (APEC), San Antonio, TX, 2018, pp. 2349-2354.

[17]Mohammad Ghanaatian, Saeed Lotfifard " Control of Flywheel Energy Storage Systems in Presence of Uncertainties" IEEE Transactions on Sustainable Energy 2018.

[18] M.S. Modarresi, L. Xie, and C. Singh. "Reserves from Controllable Swimming Pool Pumps: Reliability Assessment and Operational Planning." Proceedings of the 51st Hawaii International Conference on System Sciences. 2018. 